\title{
Synthesis and Study of PVA Based Gel Electrolyte
}

\author{
SHITAL N. BHAD* and V.S.SANGAWAR \\ Polymer Research Laboratory, P.G. Department of Physics, \\ G.V.I.S.H Amravati (M.S.)-444606, India \\ bhad_sheetal@yahoo.co.in
}

Received 13 June 2012 / Accepted 7 July 2012

\begin{abstract}
An attempt has been made to prepare polyvinyl alcohol (PVA) based proton conducting gel electrolytes in ammonium thiocyanate $\left(\mathrm{NH}_{4} \mathrm{SCN}\right)$ solution and to characterize them. XRD studies revealed that the entire gel sample are amorphous in nature with a broad peak at nearly $2 \theta=22^{0}$. Variation of conductivity of gel electrolyte with loading concentration of PVA has also been studied. It has been observed, as loading of PVA in $0.2 \mathrm{M}$ electrolyte of $\mathrm{NH}_{4} \mathrm{SCN}+\mathrm{DMSO}$ increases conductivity decreases. The conductivity of gel electrolytes exhibits liquid like nature at low polymer concentration while the behaviour is seen to be affected by the formation of $\mathrm{PVA}-\mathrm{NH}_{4} \mathrm{SCN}$ complexes upon increase in polymer content beyond $2 \mathrm{~g}$. Temperature dependence of ionic conductivity exhibits VTF and Arrhenius behaviour.
\end{abstract}

Keyword: PVA, Gel electrolyte, $\mathrm{NH}_{4} \mathrm{SCN}+\mathrm{DMSO}, \mathrm{X}-\mathrm{RD}$, Ionic conductivity

\section{Introduction}

During the last decades, there was a lot of effort done on the electrochemical preparation of polymer gel electrolytes. Polymer electrolytes have become material of great importance for use in different electrochemical devices due to their unique characteristics such as easy mouldabality, good electrode -electrolyte contact and light weight ${ }^{1,2}$. These polymer electrolytes form a bridge between liquid electrolyte and polymer. Because of their high ionic conductivity, gel electrolytes have attained precedence over conventional polymer electrolyte. In recent years, polymers such as polyvinyl alcohol (PVA), poly(vinylidene fluoride) (PVdF), polyethylene glycol (PEG), poly(vinylchloride) (PVC), poly(acrylonitrile) (PAN), poly(vinylpyrrolidone)(PVP) and poly(vinyl sulfone) (PVS) have been prominently used in development of gel polymer electrolytes ${ }^{1,3-5}$. These electrolytes have been found to posses ionic conductivity, electrochemical stability and transport properties similar to their liquid counterpart along with better dynamical properties suitable for electrochemical applications. Among the listed polymeric hosts, polyvinyl alcohol have been intensely investigated, because of its interesting properties and applications. PVA is a prominent polymer because of its good solvent holding capability and wide temperature window $^{6,7}$. Awadhia et .al. ${ }^{8}$ have reported PVA based proton conducting gel electrolyte with high ionic 
conductivity. Moreover, because of the nontoxic nature of PVA, they are particularly suitable for biomedical application like artificial tissue. Thus, in the present investigation, an attempt has been made to synthesize and characterization of PVA based gel electrolytes with ammonium salt for proton conduction.

\section{Experimental}

In the present investigation polyvinyl alcohol, ammonium thiocyanate $\left(\mathrm{NH}_{4} \mathrm{SCN}\right)$ and aprotic solvent dimethyl sulfoxide (DMSO) AR grade, s.d fine chem. Make were used for synthesis of proton conducting gel without any further purification.

\section{Synthesis}

For the synthesis of PVA gel, $30 \mathrm{~mL}$ electrolyte of $0.2 \mathrm{M}$ solution of $\mathrm{NH}_{4} \mathrm{SCN}+\mathrm{DMSO}$ was prepared. PVA was added in the electrolyte at $70{ }^{\circ} \mathrm{C}$ with constant stirring and continued for one hour maintaining the temperature. Then it was allowed to cool to room temperature so as to form the gel. PVA has been added in the electrolyte in different amount $0.5,1,1.5,2$ and 2.5 by weight (in $\mathrm{g}$ ) to form the gel of various concentration of PVA.

\section{Measurement technique}

Structural morphology of PVA gel electrolytes have been carried out by $x$-ray diffractometer. The gel electrolytes were sandwiched between two platinum electrodes and conductivity measurements were performed on 304 Systronic Conductivity Bridge at various temperatures ranging from $273 \mathrm{~K}$ to $373 \mathrm{~K}$.

\section{Results and Discussion}

\section{XRD analysis}

Figure 1 -depicts the XRD pattern for three different concentrations of PVA gel electrolytes. As seen from the Figure 1 a broad peak is noticed for the $2 \mathrm{~g}$ and $2.5 \mathrm{~g}$ PVA gel electrolytes at $2 \theta=22^{\circ}$, the slight shift of the peak is observed in $1.5 \mathrm{~g}$ PVA gel electrolyte .The broadening of XRD peaks with increasing concentration indicate enhancement in the amorphous nature of gel electrolyte ${ }^{9}$. Thus our results are in good agreement with authors ${ }^{10,11}$.

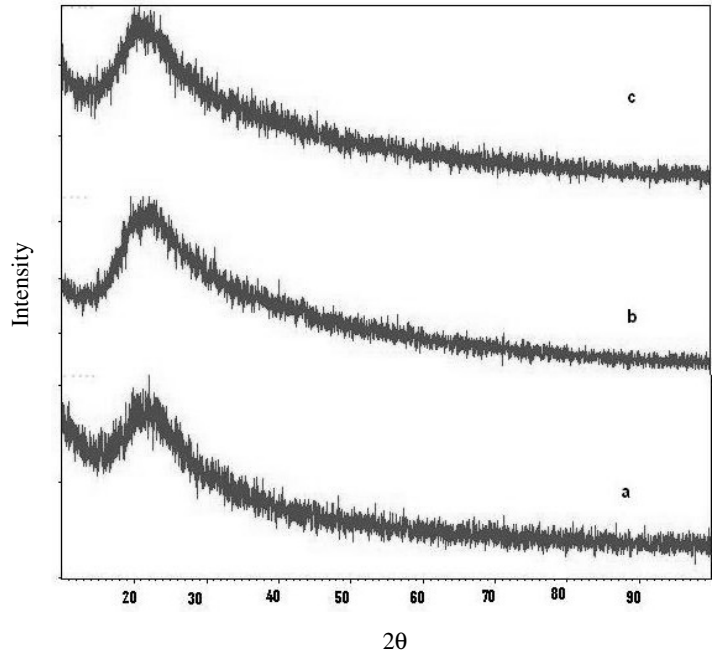

Figure 1. X-ray diffractogram of (a) $1.5 \mathrm{~g}$, (b) $2 \mathrm{~g}$ and (c) $2.5 \mathrm{~g}$ PVA gel electrolytes 


\section{Conductivity studies}

Figure 2 depicts the variation of conductivity $(\log \sigma)$ with various concentration of PVA (in $\mathrm{g}$ ) in liquid electrolyte at room temperature. It is observed that, conductivity decreases as concentration of PVA increases. This can be explained as follows

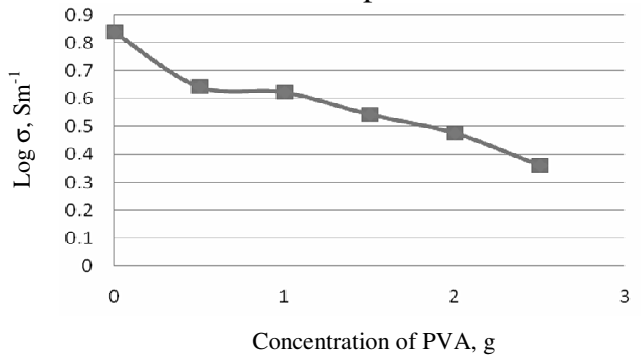

Figure 2. Variation of conductivity as a function of polymer concentration

Dimethyle sulfoxide is a highly aprotic solvent and ammonium thiocyanate is likely to dissociate in to respective ions and behaves as liquid electrolyte as reflected from high conductivity value. When polymer was added to this liquid electrolyte to form gel, the conductivity was initially seen to drop down significantly. The ionic conductivity is generally depend upon both the charge carrier concentration $(n)$ and carrier mobility $(\mu)$. As described by relation ${ }^{12}$.

$$
\sigma=n q \mu
$$

With q representing the charge of mobile carrier. Here charge carrier concentration (n), depends upon the dissociation energy $(\mathrm{U})$, involved and dielectric constant $(\varepsilon)$ as

$$
\mathrm{n}=\mathrm{n}_{0} \exp -\mathrm{U} / \varepsilon \mathrm{kT}
$$

Where, $\mathrm{k}$ is the Boltzmann constant and $\mathrm{T}$ the absolute temperature. Addition of PVA in electrolyte like $\mathrm{NH}_{4} \mathrm{SCN}$ enhances the viscosity of the system which causes a fall in mobility of charge carrier causes a significant fall in ionic conductivity of gel electrolyte .Our result are in good agreement with authors ${ }^{12,13}$.

\section{Temperature dependence conductivity}

Figure 3 shows the temperature dependence of conductivity of $0.2 \mathrm{M} \mathrm{NH}_{4} \mathrm{SCN}$ electrolyte along with PVA gel electrolytes. From the Figure 3 it is observed that for liquid electrolyte and all the samples of PVA gel electrolytes as temperature increases conductivity increases.

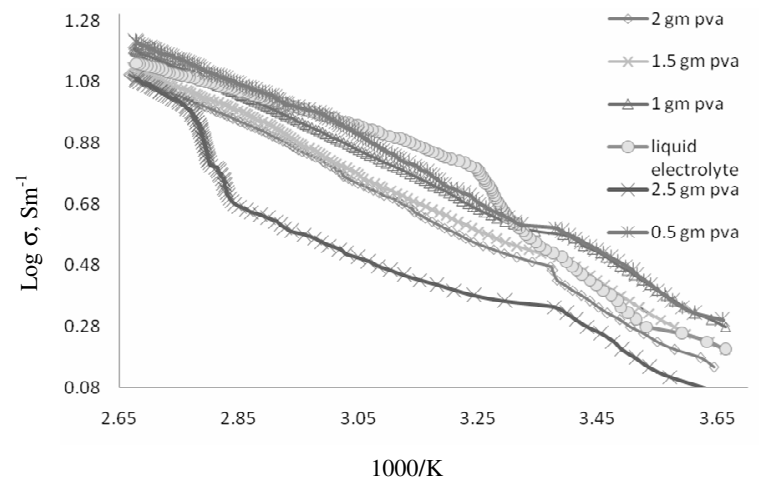

Figure 3. Conductivity as a function of temperature for liquid gel electrolyte 
The conductivity of liquid electrolyte changes with temperature this might be because of dissociation in liquid is a consequence of increasing the concentration of mobile ion with temperature is unlikely ${ }^{13}$. The PVA gel electrolytes appear to follow a behavior similar to that of liquid electrolyte particularly at lower concentration of PVA. Though the variation of conductivity with temperature for liquid electrolyte is different from that of gel electrolyte, the rate of increase in conductivity with temperature in two cases is gradual and within an order of magnitude. The conductivity of liquid electrolyte is higher than gel electrolytes. All the curves display similar behavior i.e. combination of Arrhenius and VTF behavior. Arrhenius region is noted in low temperature region, while VTF nature is exhibited at higher temperature ${ }^{14}$. The low temperature Arrhenius behavior is on the account of the presence of liquid electrolyte encapsulated by the PVA matrix. As temperature raised beyond $307 \mathrm{~K}$ segmental motion of polymer in polymer gel electrolyte comes into play and start affecting the conductivity, leading to VTF nature described by following relation ${ }^{10}$.

$$
\sigma=\sigma_{0} \exp \left(-\mathrm{B} / \mathrm{T}-\mathrm{T}_{0}\right)
$$

Where, $\sigma_{0}$ is the pre exponential factor, $\mathrm{B}$ is constant related to activation energy and $\mathrm{T}_{0}$ is reference temperature associated with ideal $\mathrm{Tg}$. The change in nature of gel electrolyte in the temperature region $289 \mathrm{~K}$ to $307 \mathrm{~K}$ is due to transition of polymorphic phase in polyvinyl alcohol. Such features are generally observed for highly amorphous polymeric system. Since the experimental PVA gel samples are found to be highly amorphous as confirmed from XRD study. The increase in conductivity with temperature is interpreted as hopping mechanism between coordinated sites, local structural relaxation and segmental motion of the polymer. As the amorphous region progressively increases, however, the polymer chain acquires faster internal motion and bond rotations. This in turn favors the hopping of inter-chain and intra-chain movement and ionic conductivity of polymer electrolyte becomes high.

Besides exhibiting VTF behavior the temperature dependence of conductivity also depicts significant variation for the gel electrolyte containing higher amount of PVA i.e. beyond $2 \mathrm{~g}$ of PVA, on account of formation of PVA $-\mathrm{NH}_{4} \mathrm{SCN}$ complex. Our results are in good agreement with Shukla et al. ${ }^{7}$, Patel ${ }^{10}$, Sekhon et al. ${ }^{15}$, Chand et. al. ${ }^{16}$ and Pandey et. al ${ }^{17}$.

\section{Conclusion}

Polymer gel electrolytes prepared by addition of PVA in $0.2 \mathrm{M} \mathrm{NH}_{4} \mathrm{SCN}$ in DMSO have been structurally and electrically characterized in the present work. XRD patterns exhibit broadening of peak in diffraction patterns for $2.5 \mathrm{~g}$ PVA gel electrolyte indicating increase in amorphousness. Gel of higher concentration of PVA shows the presence of PVA: $\mathrm{NH}_{4} \mathrm{SCN}$ complex and conductivity is seen to be influenced by the presence of this complex. Temperature dependence of ionic conductivity of gel electrolyte reflects Arrhenius and VTF behaviour.

\section{References}

1. Gilbert M, Polymer Rev., 1994, C34(1), 77-135.

2. Gray F M, Solid Polymer electrolyte - fundamental and technological application, New York: VCH, 1991.

3. Awadhia A and Patel S K, Progress in Crystal Growth and Characterization of Materials, 2006, 52(1-2), 61-68.

4. Patel S K, Awadhia A and Agrawal S L, Phase Transition, 2009, 82(6),.421-432.

5. Ueberschlag P, Sensor Rev., 2001, 21(2), 118-126. 
6. Finch C A, (Ed.,) Polyvinyl 1 alcohol: properties and applications Bristol: John Wiley \& Sons, 1973.

7. Shukla P K and Agrawal S L, Indian J Pure Appl Phys., 2000, 38, 53

8. Awadhia A and Agrawal S L, Solid State Ionics, 2007, 178, 951

9. Agrawal S L and Shukla P K, Phys Stat Sol (a), 1997, 163, 247-254

10. Patel S K, Patel R.B, Awadhia A, Chand N and Agrawal S L, Pramana J Phys., 2007, 69(3), 467.

11. Shukla P K and. Agrawal S L, Ionics, 2000, 6, 312 .

12. Deepa M, Sharma N, Varshney P, Chandra R and Agnihotry S A, Ion Conducting Materials:Theory and Application (Eds.,) Kulkarni A R and Gopalan P, New Dehali: Narosa Publishing House, 2001, 74

13. Agrawal S L and Awadhia Arvind, Bull Mater Sci., 2004, 27(6), 523-527.

14. MacCallum J R and Vincent C A, (Eds.), Polymer Electrolyte Reviews, London: Elservier Applied Sciences Publisher, 1989, 1-2.

15. Sekhon S S, Bull Mater Sci., 2003, 26, 321.

16. Chand N, Rai N, Agrawal S L and Patel S K, Bull Mater Sci., 2011, 34(7), 1297-1304.

17. Pandey K, Dwivadi M M and Asthana N, Mater Sci Appl., 2011, 2, 721-728.

18. Abraham K M and Alamgir M, J Electrochem Soc., 1990, 137, 1657-1658.

19. Feuillade G and Perche P H, J Appl Electorchem., 1975, 5, 63.

20. Shukla P K and Agrawal S L, Bull Electrochem., 1996, 12, 732

21. Scrosati B and Vincent C A, MR S Bull., 2000, 25, 28.

22. Sekhon S S, Pradeep and Agnihotry S A, Solid State Ionics: Science and Technology (Eds.,) Chowdari B V R, et al, Singapore : World Scientific ,1998 217.

23. Webber A, J Electrochem Soc., 1991, 138, 2586. 